\title{
INTELLIGENT LEARNING OBJECTS
}

An Agent-Based Approach of Learning Objects

\author{
Ricardo Silveira, Eduardo Gomes and Rosa Vicari \\ Universidade Federal de Pelotas, - UFPEL, Campus Universitário, s/n ${ }^{\circ}$ - Caixa Postal 354 \\ Pelotas-RS-Brazil rsilv@ufpel.edu.br \\ Universidade Federal do Rio Grande do Sul Av. Bento Gonçalves, 9500 - Campus do Vale - \\ BlocoIV Porto Alegre-RS-Brazil.ergomes@inf.ufrgs.br rosa@inf.ufrgs.br
}

\begin{abstract}
Many people have been working hard to produce metadata specification towards a construction of Learning Objects in order to improve efficiency, efficacy and reusability of learning content based on an Object Oriented design paradigm. The possibility of reusing learning material is very important to designing learning environments for real-life learning. At the same time, many researchers on Intelligent Learning Environments have proposed the use of Artificial Intelligence through architectures based on agent societies. Teaching systems based on Multi-Agent architectures make it possible to support the development of more interactive and adaptable systems. This paper proposes an agent-based approach to produce more intelligent learning objects (ILO) according to the FIPA agent architecture reference model and the LOM/IEEE 1484 learning object specification.
\end{abstract}

Key words: Learning Objects, Real Life Learning Environments, Artificial Intelligence.

\section{INTRODUCTION}

This paper addresses the integration between Learning Objects and Multi-Agent Systems. A Learning Object according to Downes (2001), (2002), Mohan and Brooks (2003), Sosteric and Hesemeier (2002), is an entity of learning content which can be used several times in different courses or in different situations. The use of reusable learning objects to create learning environments improves quickness, flexibility and economy. According to Downes (2001), the cost of developing learning materials for on line learning can be large, but as the content of related courses taught at different universities and organizations often tend to be similar, the cost of 
developing the learning material can be shared among its potential users. The object learning approach promises to reduce significantly the time and the cost required to develop on line courses. A specific content can be used for different courses, in different places, and for different purposes.

A learning object must be modular, discoverable and interoperable, in order to be reused. To achieve these features and improve efficiency, efficacy and reusability of learning objects, many people have dedicated long hours of hard work. The majority of the efforts focus on the definition of standardization. Organizations such as IMS Global Learning Consortium (2004), IEEE (2004), ARIADNE (2004), and CanCore (2004), have contributed significantly by defining indexing standards called metadata (data about data). Metadata structures contain information to explain what the learning object is about, how to search, access, and identify it and how to retrieve educational content according to a specific demand.

These features of learning objects are very useful in designing learning environments for real-life learning applications. However, Downes (2002) points out that a lot of work must be done in order to use a learning object. First, it is necessary to build an educational environment in which they can function. It also becomes necessary to locate these objects and then arrange them in their proper order according to their design and function. In certain cases - as for example when the object is a Flash animation or a chunk of streaming media, you must arrange for the installation and configuration of appropriate viewing software, and the objects must be delivered in some appropriate context like a problem solving environment. Although all of this seems to be easier to deal with, we need smarter learning objects.

Mohan and Brooks (2003) point out several limitations of current learning objects. According to them, an instructional designer must carefully examine each learning object in which the task of finding the right object may be quite time consuming. In addition, the current learning object metadata standards are not very useful to support pedagogical decisions.

In order to improve the use of learning objects in real-life learning we propose the convergence of this technology with Artificial Intelligence. The state of the art in Intelligent Tutoring Systems (ITS) and Intelligent Learning Environments (ILE) fields points to the use of Agent Society-Based Architectures. The fundamentals of the multi-agent systems have been demonstrated to be very appropriate to designing tutoring systems, since the teaching-learning problem could be handled in a cooperative approach (Johnson \& Shaw, 1997). Using a multi-agent systems approach to designing intelligent tutoring systems can result in faster, more versatile and low costs systems.

We believe that the convergence between learning objects and multiagent systems can produce Intelligent Learning Objects (ILO). We propose a 
learning object with agent characteristics, such as autonomy, knowledge about itself and its environment, sociability and goals. Such a learning object can be more useful than current learning objects and can help to overcome some limitations presented above.

\section{WHY TRANSFORM LEARNING OBJECTS IN AGENTS?}

There are many benefits in integrating learning objects and agents. An Intelligent Agent is a software entity that works in a continuous and autonomous way in a particular environment, generally inhabited by other agents, and able to interfere in that environment, in a flexible and intelligent way, not requiring human intervention or guidance (Bradshaw, 1997). An agent is able to communicate with others by message exchange using a high level communication language called Agent Communication Language (ACL), which is based on the Speech Act Theory (Searle, 1981).

The most complete current learning object model, the SCORM used in real-life learning (see next section), performs the communication by calling methods (functions) and passing parameters, according to the Object Oriented Programming paradigm. Using ACL for communication among learning objects, the learning environments can perform a more powerful communication rather than this approach.

With an ACL, it is possible to supply a more powerful semantic in communication using a formal protocol and a formal Content Language (CL) based on some logic formalism to express the messages content. Using an ACL the learning objects can communicate not only variable values, but also facts, rules, mental states and others. The result is that communication using ACL and CL is potentially better than communication using the object oriented approach, like the current learning object models do.

Another interesting useful capability of intelligent agents is their potential learning ability. This feature gives to an ILO the ability to acquire new knowledge and perform different behaviours during its existence, according to its own experience. Thus, by interaction with students and other ILO, the ILO is able to evolve. It is not static like current learning objects.

The possibilities are great: an ILO can summon others to promote a learning experience that can help students in specific points; get students preferences and adapt the content presentation with the student; perform sophisticated evaluation methods, and others.

There are also many researchers addressing coordination and cooperation mechanisms among agents that help the agent society to achieve its goals. Such work can be very useful due to the possibility of a self-organizing ILO 
society where it can promote richer learning experiences. The coordination and cooperation mechanisms enable complex behaviours and interactions among the ILO and, as a consequence, more powerful learning experiences.

Some types of agents deliberate and make plans based on a set of mental states. These types of architecture, called BDI (Belief, Desire and Intention Shohan 1993), can be very useful to model complex behaviours. The ILO modelled through BDI architectures can implement advanced learning objects.

Other agent features that can be interesting are autonomy, pro-activity, sociability and benevolence. The autonomy of an ILO gives it the capability to act based on its own behaviour and knowledge with no external intervention. The pro-activity feature assures that the ILO must act in order to satisfy its own objectives. The sociability and benevolence features address the capability of social and cooperative behaviour.

\section{DEVELOPING THE INTELLIGENT LEARNING OBJECTS APPROACH}

Some researchers concerning learning objects, (Mohan \& Brooks, 2003) propose an object-oriented approach. This architecture has a class of objects called LearningObject, which is the superclass of all learning objects. Every learning object is an instance of this class.

The Sharable Content Object Reference Model (SCORM®, 2004) is currently the most complete model of reference for learning content reuse, and it is very useful in real-life learning. In the SCORM model, the learning resources (assets) are added in packages (SCO). Each SCO has an archive (manifest file) with information about the content that it loads and on how it must be used. Besides such information are the SCO metadata and the assets metadata. There are also references for assets and sequencing rules.

Communication between the SCO and a Learning Management System (LMS) is made through a function call of an Application Program Interface (API) implemented for the LMS. These calls are made in JavaScript and can involve information about the students. This information is stored in a Data Model, which allows one standardized communication between SCOs and LMSs. To construct an SCO means to build the manifest file and add together all the learning resources in an archive. The SCO must be able to call upon the API methods.

To follow the SCORM run-time model, an Intelligent Learning Object must be able to manipulate information such as metadata, references to learning resources and rules for sequencing these learning resources. They 
must have a way of communicating between Intelligent Learning Objects and LMSs.

To achieve agent capabilities the Intelligent Learning Objects must communicate through message exchange using an Agent Communication Language (ACL) and a Content Language (CL). The Foundation of Intelligent Physical Agents (FIPA, 2002), a non-profit organization, produced standards for the inter-operation of heterogeneous software agents. Two important contributions of FIPA are an agent communication language called FIPA-ACL and a content language called FIPA-SL. There are several frameworks that allow the construction of FIPA complaint agents. They provide a set of JAVA classes and services for communication between the agents. Thus, we will use one of them as a reference to implement the Intelligent Learning Object model.

\section{INTELLIGENT LEARNING OBJECTS MULTI- AGENT ARCHITECTURE}

The proposed multi-agent architecture (Fig 1) encompasses two types of agents: Intelligent Learning Objects and LMS agent. The LMS agent represents all the features of the learning environment which are not performed by ILO. The Intelligent Learning Objects are our object of study in this work. The agent environment that these agents inhabit is a FIPA complaint environment provided by FIPA Operational System (FIPA-OS) Framework (FIPA-OS 2002).

Figure 1. Intelligent Learning Objects multi-agent architecture.

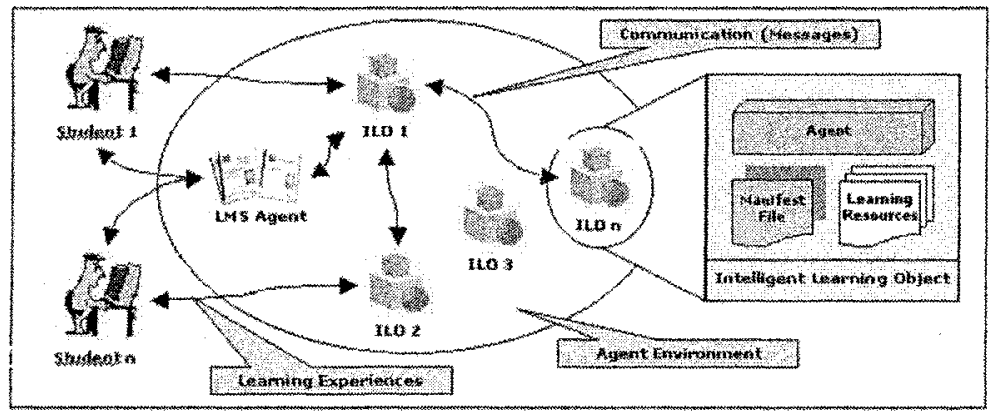

Students interact with an LMS agent in order to gain learning experiences. The LMS agent searches for the appropriate ILO and invokes it. The ILO is then is then responsible for generating learning experiences for the students. In this task, it can communicate with the LMS agent along with 
other agents in order to promote richer learning experiences. All the communication is performed by message exchange in FIPA-ACL.

The LMS agent task is to manage student interactions with the learning environment where the ILO society is working. Thus, it provides a way for the students access to the ILO, stores information about the students, and passes to the ILO all the student information. The LMS agents must be able to communicate with the Intelligent Learning Objects.

An Intelligent Learning Object is an agent that is able to play the role of a learning object. However, it is also a special type of learning object that contains agent features. These two definitions are correct. The responsibility of the ILO is generating learning experiences to the students. The ILOs are agents that can generate learning experiences in the sense of learning objects, that is, they must be modular, discoverable, inter-operable, and, most important of all, reusable.

\section{AGENT ARCHITECTURE FOR AN ILO}

The Intelligent Learning Object model is composed of an agent, a Manifest File, and Learning Resources. The agent is able to interpret the Manifest File, which has information about how the agent should work. The Manifest File contains references to learning resources (content packaging information). Learning resources are actual files containing learning content. The ILO is able to deliver these learning resources to students. The Manifest File also has information on how the learning resources should be delivered to students (Sequence Rules) and about what the learning experience generated by the ILO is about (Metadata). The internal architecture of this type of Intelligent Learning Object is as shown in Figure 2.

Figure 2. Internal architecture of ILO

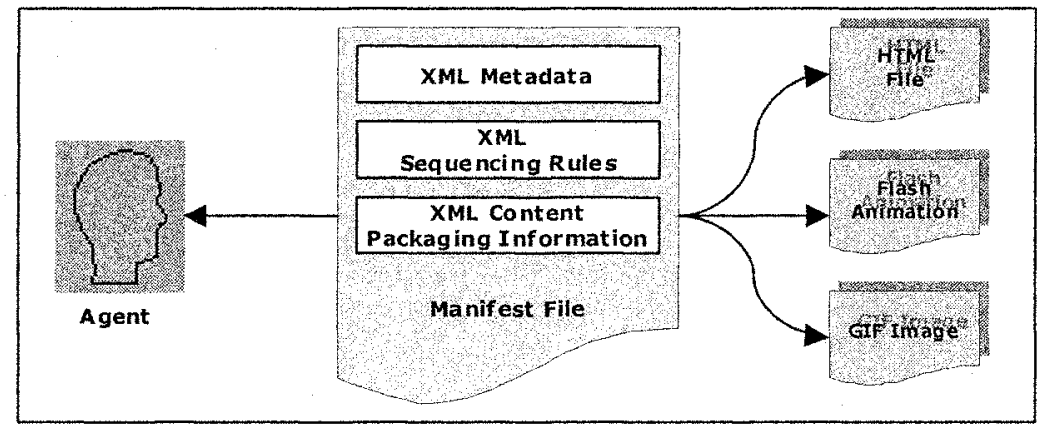

The metadata information is used to describe the learning content that the Intelligent Learning Object loads. Metadata information enables the search and discoverability of the ILO model. The most complete current metadata 
model to describe learning content is the P1484.12.1 IEEE Standard for Learning Object Metadata (IEEE 2004), an adopted metadata model. The metadata information is described in XML using the P1484.12.3 Standard for XML binding for Learning Object Metadata data model (IEEE 2004).

The content packaging information provides the functionality to describe and pack learning materials. Content packaging addresses the description, structure, and location of the learning resources and the definition of some particular content types. The content packaging uses the IMS Content Packaging Information (IMS 2004). This is described in XML using the IMS Content Packaging XML Binding Specification.

Sequencing rules define a method for representing the intended behaviour of an authored learning experience so that the ILO can sequence the Learning Resources. The specification defines the required behaviours and functionality that a conforming ILO must implement. It incorporates rules that describe the branching or flow of instruction through content according to the outcomes of a learner's interactions with content. We are using the IMS Simple Sequencing Information and Behaviour Model described through the IMS Simple Sequencing XML Binding (IMS 2004).

The Learning Resources represent the actual files referenced by the content packaging information. These files may be local files that are contained within the ILO, or they can be external files that are referenced by a Universal Resource Indicator. They can be of any type that can be represented in a Web Browser. This possibility is very important for distance learning applications.

\section{CONCLUSIONS}

At this point, we quote Downes (2002): We need to stop thinking of learning objects as chunks of instructional content and to start thinking of them as small, self-reliant computer programs. This means more than giving a learning object some sort of functionality, more than writing Java calculators or interactive animations. When we think of a learning object we need to think of it as a small computer program that is aware of and can interact with its environment.

Intelligent Learning Objects are able to improve the adaptability and interactivity of complex learning environments built with these kinds of components by the interaction among the learning objects and between learning objects and other agents in a more robust conception of communication rather than a single method invocation as the object-oriented paradigm use to be. 


\section{REFERENCES}

Advanced Distributed Learning (ADL)a. (2004) Sharable Content Object Reference Model (SCORM (B) 2004 Overview. [www.adlnet.org].

Alliance of remote instructional authoring \& distribution networks for Europe. (2004), ARIADNE, [http://ariadne.unil.ch].

Bradshaw, J. M. (1997)An introduction to software agents In: Bradshaw, J. M. Ed. Software Agents. Massachusetts: MIT Press, 1997.

CanCore (2004) Canadian Core About. [http:/www.cancore.ca/about.html].

Downes, S. (2001), Learning objects: resources for distance education worldwide. in International Review of Research in Open and Distance Learning, 2(1). 2001

Downes, Stephen (2002) Smart Learning Objects.

FIPA: The foundation for Intelligent Physical Agents (2002). Specifications. [http://www.fipa.org\}..

IEEE Learning Technology Standards Committee (2004) Specifications. [ http://ltsc.iee.org].

IMS Global Learning Consortium. (2004) Current specifications. [http://www.imsglobal.org /specifications.cfm].

Johnson, W. Lewis; Shaw, Erin. (2000) Using agents to overcome deficiencies in web-based courseware. In: World Conference on Artificial Intelligence in Education, AI-ED, 8., 1997. Proceedings... [www.isi.edu/isd/johnson.html.].

Mohan, P.and Brooks, C. (2003) Engineering a Future for Web-based Learning Objects. Proceedings of International Conference on Web Engineering, Oviedo, Asturias, Spain.

Searle, John R. (1981) Speech Acts - An Essay in the Philosophy of Language. Coimbra: Livraria Almedina.

Shoham, Y (1993) Agent-oriented programming. Artificial Intelligence, Amsterdam, n.60, v. 1, p. $51-92$.

Sosteric, Mike, Hesmeier, Susan (2002) When is a Learning Object not an Object: A first step towards a theory of learning objects International Review of Research in Open and Distance Learning ISSN: 1492-3831

\section{ACKNOWLEDGEMENT}

This project is granted by Brazilian research agencies: CNPq and FAPERGS. 